Recepción: 20 / 04 / 2017

Aceptación: 20 / 05 / 2017

Publicación: 15 / 07 / 2017

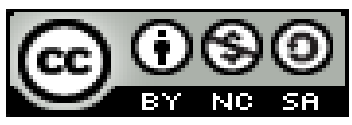

Articulo de investigación

\title{
Síndrome de Danny Walker: Presentación de un caso clínico
}

\author{
Dandy Walker syndrome: a case report
}

\section{Síndrome de Dandy Walker: Apresentação de um caso clínico}

Aracelly P. Parrales-Cedeño ${ }^{\text {I }}$ dra_parrales@yahoo.com

Arcelia V. Tenemaza-Valladares II arce.tenemaza@gmail.com

Patricia A. Palacios-Parrales III patriciapp@gmail.com

Correspondencia: dra_parrales@yahoo.com

\footnotetext{
I. Coordinadora de la Gestión de Emergencia. Hospital Pediátrico Dr. Francisco de Icaza Bustamante. Docente de Pediatría, Facultad de Ciencias Médicas, Escuela de Medicina; Universidad de Guayaquil, Guayaquil, Ecuador.

II. Médico Residente Asistencial del Hospital Pediátrico Dr. Francisco de Icaza Bustamante; Universidad de Guayaquil, Guayaquil, Ecuador.

III. Médico Postgrado de Pediatría; Universidad Católica Santiago de Guayaquil, Guayaquil, Ecuador.
} 


\section{Resumen}

El síndrome de Dandy-Walker es una anomalía cerebral congénita caracterizada por hipoplasia del vermis cerebeloso, dilatación quística del cuarto ventrículo y fosa posterior agrandada. El objetivo de este artículo es describir el caso de una enfermedad rara, haciendo énfasis en que puede haber subdiagnóstico; ya que se adjudica la etiología de las alteraciones neurológicas erróneamente a asfixia neonatal. Se trata de una niña de 3 años con antecedentes de hipoxia neonatal y convulsiones desde los 7 meses de edad, que llega a hospital con movimientos tónico-clónico generalizados, que se controla con anticonvulsivantes, presentando retraso del desarrollo madurativo para su edad, no habla, hipotonía generalizada. Se le realiza Tomografia Computarizada y Resonancia Magnética de Cerebro que mostró disminución del volumen del cerebelo, con contenido de intensidad líquida extra-axial en fosa posterior con comunicación al cuarto ventrículo, dilatación de los ventrículos laterales y ausencia de cuerpo calloso. Se cataloga al caso presentado como un Síndrome o Malformación de Dandy Walker tanto por la sintomatología clínica como radiológica expuesta.

Palabras claves: Síndrome de Dandy-Walker; alteraciones neurológicas; hidrocefalia. 


\section{Abstract}

Dandy-Walker syndrome is a congenital brain anomaly characterized by cerebellar vermis hypoplasia, cystic dilatation of the fourth ventricle, and enlarged posterior fossa. The aim of this article is to describe the case of a rare disease, emphasizing that there may be underdiagnosis; Since the etiology of the neurological alterations is erroneously attributed to neonatal asphyxia. It is a 3 year-old girl with a history of neonatal hypoxia and seizures since the age of 7 months, who arrives at the hospital with generalized tonic-clonic movements, controlled by anticonvulsants, presenting developmental delay for her age, Generalized hypotonia. Computed Tomography and Magnetic Resonance of the Brain were performed, showing a decrease in cerebellum volume, with an extraaxial fluid content in the posterior fossa with communication to the fourth ventricle, dilation of the lateral ventricles, and absence of corpus callosum. The case presented as a Syndrome or Malformation of Dandy Walker is cataloged by both clinical and radiological symptoms exposed.

Key words: Dandy-Walker syndrome; neurological disorders; hydrocephalus. 


\section{Resumo}

A síndrome de Dandy-Walker é uma anomalia congénita caracterizada por hipoplasia cerebral do vermis cerebelar, dilatação cística do quarto ventrículo e da fossa posterior alargada. O objetivo deste artigo é descrever o caso de uma doença rara, enfatizando que podem ser pouco; porque a etiologia de doenças neurológicas erroneamente atribuído alterações asfixia neonatal. É uma menina de 3 anos com uma história de hipóxia neonatal e convulsões desde que ele foi de 7 meses de idade, que vem ao hospital com movimentos tônico-clônicas generalizadas, que é controlada por anticonvulsivantes, apresentando atraso no desenvolvimento maturacional para sua idade, não fala , hipotonia generalizada. Submeteu-se a tomografia computadorizada e ressonância magnética do cérebro mostrou diminuição do volume cerebelar, contendo extra-axiais de corrente na fossa posterior líquido com comunicação para o quarto ventrículo dilatação dos ventrículos laterais e ausência do corpo caloso. Ele lista o caso apresentado como uma síndrome síndrome ou DandyWalker ambos os sintomas clínicos e radiológicos exposta.

Palavras-chave: Síndrome de Dandy-Walker; distúrbios neurológicos; hidrocefalia. 


\section{Introducción}

El síndrome de Dandy-Walker conocida actualmente como Malformación de Dandy-Walker, se trata de una anomalía cerebral congénita caracterizada por hipoplasia del vermis cerebeloso, dilatación quística del cuarto ventrículo y fosa posterior agrandada. (Treviño, 2014)

Es una enfermedad rara, cuya prevalencia en los Estados Unidos es de 1 caso por cada 35.000 nacidos vivos, con predominio en mujeres sobre hombres (en relación 3:1) (Cueva, 2016). Su etiología permanece incierta aunque algunas cromosomopatías se asocian entre el 17,6\% y el 54\%, encontrándose con mayor frecuencia las trisomías 13, 18 y 21; por lo que resulta importante la genealogía familiar especialmente en casos de retardo mental, retraso en el desarrollo, malformaciones congénitas, abortos, mortinatos y muerte infantil que oriente hacia algún tipo de herencia para la evaluación de estas enfermedades con expresividad variable. (Dandy-Walker Alliance, 2008)

Las manifestaciones clínicas son variadas e inespecíficas, y están relacionadas directamente con las alteraciones anatómicas, mientras más severas sean éstas mayor es la sintomatología. Un factor importante que incide sobre la clínica es la edad y malformaciones asociadas al momento del diagnóstico. Los síntomas pueden incluir irritabilidad, convulsiones, nistagmus, vómitos, retardo en el desarrollo psicomotriz, alteración del lenguaje, aumento del perímetro cefálico (hidrocefalia), dilatación y congestión de venas del cuero cabelludo, abombamiento de la fontanela anterior y diástasis de suturas craneales. (Figueroa, 2013). Los síntomas que suelen presentarse en la primera infancia incluyen lentitud del desarrollo motor y aumento progresivo del tamaño del cráneo. (Nacional Institute of Neurological Disorders and Stroke, 2008) 
Esta malformación de Dandy-Walker se diagnostica por neuroradiología que incluye ultrasonido, resonancia magnética nuclear (RM) y tomografía computarizada (TC). Inicialmente la evaluación debe hacerse con ultrasonido, que es el método de elección para el diagnóstico prenatal mediante ultrasonografía transvaginal a partir de la semana 18 de gestación, momento en el cual el vermix cerebeloso debiera estar formado completamente, antes no. (Wald, 2004). En la actualidad la RM es considerada la herramienta diagnóstica más precisa para el diagnóstico de Dandy Walker puesto que permite una delimitación anatómica más precisa sea para con fines diagnósticos o de tratamiento. (Blaas, 2008)

El tratamiento depende de la sintomatología; una derivación entre el quiste y la cavidad peritoneal está indicada ante síntomas de hipertensión endocraneal, y será necesaria la colocación de un drenaje ventriculoperitoneal o ventriculoauricular, en los casos en que la sintomatología continúe. (Figueroa, 2013). En los últimos años se ha probado nuevos métodos quirúrgicos, como la ventriculostomía endoscópica del tercer ventrículo, que contribuye a una disminución ligera en el tamaño ventricular y reducción, en grado variable, del quiste. Concomitantes a estos procedimientos quirúrgicos, se requiere un manejo sintomático, con rehabilitación psicomotora y terapia del lenguaje. (Spennato, 2011)

En lo referente al pronóstico, es variable puesto que depende de las malformaciones asociadas. Así, es moderadamente favorable si no se asocia con otras malformaciones congénitas y si la hidrocefalia se trata oportuna y adecuadamente. (Zuluaga, 2009). El síndrome de Dandy Walker como anormalidad aislada tiene alta posibilidad de supervivencia, se ha descrito supervivencia del 15\% de los pacientes que fueron diagnosticados en el embarazo y con síntomas en edad temprana y hay reportes de personas que han tenido este diagnóstico durante toda su vida sin ningún síntoma. (Martínez, 2009) 


\section{Materiales y métodos}

\section{Caso clínico:}

Paciente femenino de 3 años de edad, que ingresa a hospital pediátrico con cuadro clinico caracterizado por movimientos tónico clónicos generalizados, oculogiros, sialorrea, relajación de esfínteres de aproximadamente 30 minutos de duración.

Dentro de los antecedentes personales se destaca que es producto de quinto embarazo obtenido por parto vaginal intradomiciliario con datos de hipoxia neonatal e historia de episodios convulsivos desde los 7 meses de edad, hospitalizado en 3 ocasiones donde se implementó tratamiento con fenitoina y ácido valproico. No se reporta antecedentes familiares de retraso mentalo epilepsia, tampoco abortos ni mortinatos.

Al examen físico de su ingreso presentaba signos de desnutrición moderada con peso de $10 \mathrm{~kg}$ (debajo de percentil -2 peso para la edad según patrones de crecimiento OMS) y talla de $85 \mathrm{~cm}$ (debajo de percentil -2 talla para la edad según patrones de crecimiento OMS), frecuencia cardiaca 120 latidos por minuto, frecuencia respiratoria 30 respiraciones por min, temperatura de 36.8 grados centígrados. Presencia de macrocefalia con un perímetro cefálico de $52 \mathrm{~cm}$ (encima de percentil +2 perímetro cefálico para la edad según patrones de crecimiento OMS), retraso del desarrollo madurativo para su edad: no sostén cefálico, no se sienta, no camina, no sujeta objetos, no tiene control de esfínteres, no vocaliza palabras sólo emite sonidos, no se ríe, no juega, no come por sí sola, hipotonia generalizada.

Las convulsiones a su ingreso fueron controladas con diazepan, luego se realizó impregnación con fenitoína y se continuó con dosis de mantenimiento más un plan de vigilancia para realizar estudios 
complementarios. Dentro de los exámenes se realizó Tomografia simple de cerebro que reveló Cuarto ventrículo abierto hacia atrás, Ventrículos laterales dilatados y ausencia de cuerpo calloso.

En la Resonancia Magnética se observó disminución del volumen del cerebelo, con contenido de intensidad líquida extra-axial en fosa posterior que comunica con el cuarto ventrículo. Disminución del volumen del tronco encefálico. Dilatación de los ventrículos laterales con alteración de la morfología de línea media. No se observa cuerpo calloso.

Durante el cuarto día permaneció estable, sin presentar nuevos episodios convulsivos, por lo que tras vigilancia por $48 \mathrm{~h}$ adicionales fue dado de alta con indicación de seguimiento y control por neurología pediátrica.

A los 7 meses de seguimiento neurológico el paciente continuó con su tratamiento anticonvulsivante y no volvió a presentar crisis convulsivas; además continuó en manejo por un nutricionista y un gastroenterólogo pediatra para mejorar su estado nutricional

\section{Resultados}

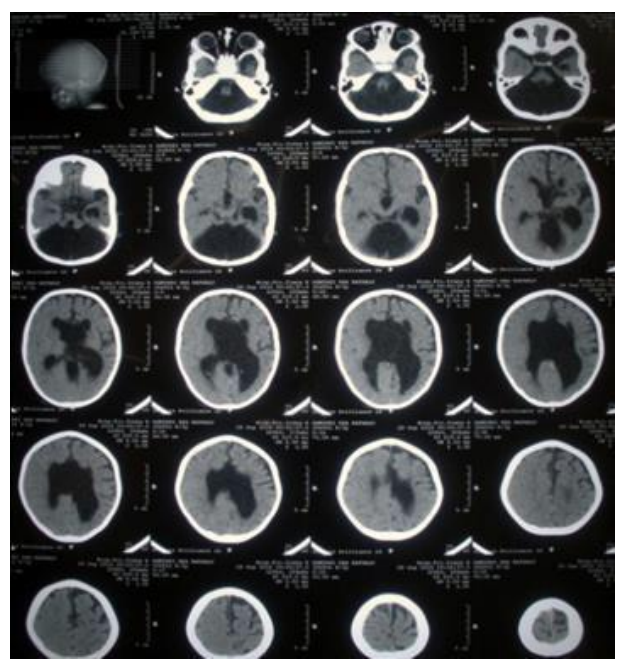

TAC Simple de Cerebro: 
Cuarto ventrículo abierto hacia atrás. Ventrículos laterales dilatados. No se visualiza cuerpo calloso. Mastoides neumatizadas.
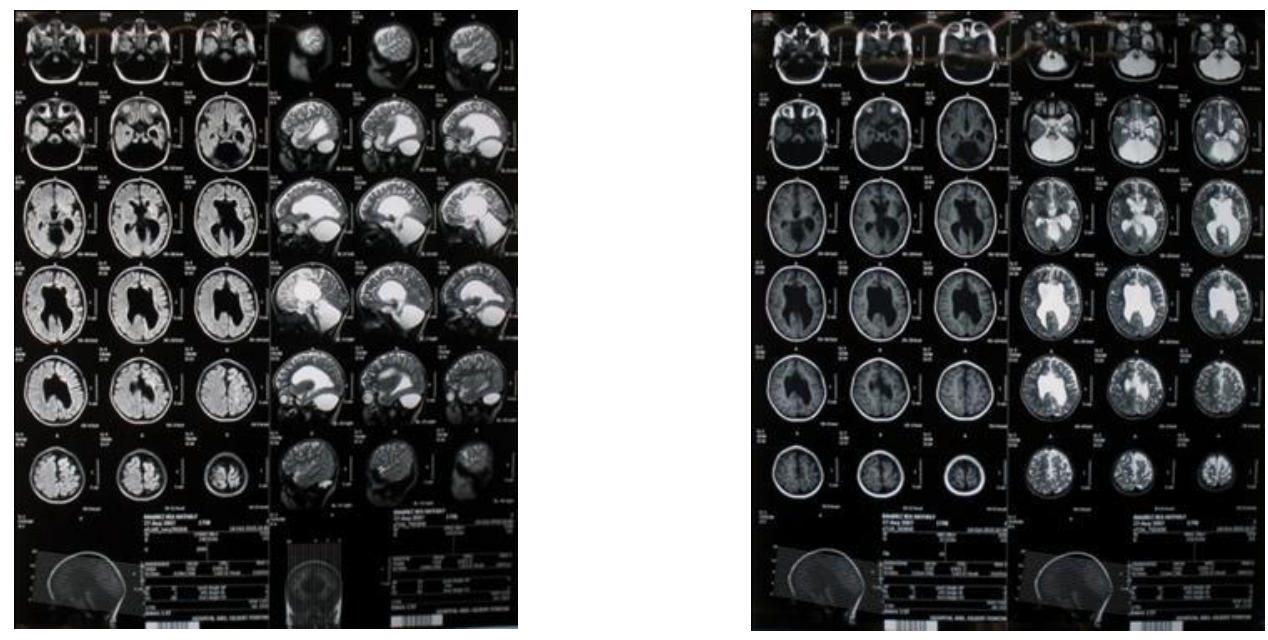

\section{RMN Cerebro}

Se observa disminución del volumen del cerebelo, con contenido de intensidad líquida extra axial en fosa posterior que comunica con el cuarto ventrículo. Disminución del volumen del tronco encefálico. Dilatación de los ventrículos laterales con alteración de la morfología de línea media. No se observa cuerpo calloso.

\section{Discusión.}

El Síndrome de Dandy-Walker es una anomalía congénita adquirida durante el período embrionario, que se caracteriza principalmente por alteraciones en el desarrollo del vermix cerebeloso, dilatación quística del cuarto ventrículo, presencia de hidrocefalia lo que produce un agrandamiento de la fosa posterior y que suele aparecer en la infancia. (Rodríguez, 2010). La literatura menciona una incidencia de un caso en 35.000 recién nacidos vivos, con predominio en el sexo femenino (Cueva, 2016). Lamentablemente en el Ecuador no se ha encontrado datos exactos de la incidencia, 
prevalencia y tasa de morbimortalidad de este síndrome, sin embargo, en ocasiones puede haber subdiagnóstico; ya que se adjudica la etiología de las alteraciones neurológicas erróneamente a asfixia neonatal, como ocurre en el caso presentado, y en realidad se trata de una malformación prenatal.

Las alteraciones neurológicas que se presentan especialmente en el primer año de vida, sus causas debe ser investigadas especialmente la causa genética, siendo importante los antecedentes ginecoobstetricos de la madre como abortos, mortinatos, aunque en este caso no existen antecedentes de este tipo, asi como tampoco antecedentes familiares de retardo mental. (Figueroa, 2013)

Las manifestaciones clínicas, si bien son inespecíficas, en su gran mayoría los afectados presentan síntomas durante el primer año de vida, como convulsiones, retraso en el desarrollo psicomotor, dificultades visuales y auditivas, hipotonía, entre otros (National Institute of Nurological Disorders and Stroke, 2008). En nuestro paciente la clínica concuerda con la literatura consultada, que refiere que aproximadamente el $90 \%$ presenta síntomas en el primer año de vida.

El diagnóstico de esta patología es a través de técnicas neuroradiológicas que incluyen: ultrasonido, resonancia magnética $(\mathrm{RM})$ y tomografía computarizada (TC); siendo el ultrasonido el método de elección para el diagnóstico prenatal (Treviño,2014). En el caso descrito, el diagnóstico se hizo a los 3 años de edad, mediante TC y RM de Cerebro, que informaron: hidrocefalia, dilatación ventricular, cerebelo pequeño, y agenesia del cuerpo calloso, hallazgos que se asocian con el Síndrome de Dandy Walker. Desafortunadamente no hubo evaluación en período prenatal.

El tratamiento se dirige a los síntomas, como derivación del ventrículo peritoneal en caso de hipertensión endocraneal (Spennato, 2011). En nuestro paciente se lograron controlar las crisis convulsivas con fenitoina estabilizándose su epilepsia, a más de contar con un programa de 
rehabilitación con terapia de ejercicios de motricidad y apoyo de nutricionista para soporte de estado nutricional.

\section{Conclusiones.}

Se cataloga al caso presentado como un Síndrome o Malformación de Dandy Walker tanto por la sintomatología clínica como radiológica expuesta.

La malformación de Dandy-Walker es una patología rara en el mundo y en nuestro medio existen pocos casos publicados, no existiendo estadísticas nacionales sobre la enfermedad.

\section{Bibliografía.}

Blaas H, Eik-Nes SH. First-trimester, diagnosis of fetal malformations. In: Rodeck CH, editor. Fetal medicine. 2. ed. Philadelphia: Churchill Livingstone; 2008. p. 587-8

Cueva, J., Lozano, A., Irias, M., Vásquez, R., Varela, D., Variante de Dandy Walker: reporte de un caso. $\square$ Rev Chil Pediatr. 2016;87(5):406-410

Dandy-Walker Alliance. Maryland: Dandy-Walker Alliance; 2008. Disponible en: http://www.dandywalker.org/index_es.html

Figueroa V., Reyes, I., García, R., Rodriguez C., Martínez, I., Síndrome de Dandy-Walker. Arch Neurocien (Mex) Vol. 18, No. 2: 92-98; 2013

Martínez, N., Elizondo, A., Variante del síndrome de Dandy Walker. Diagnóstico y consejo prenatal. 2009;52:124-7.

National Institute of Neurological Disorders and Stroke. Dandy-Walker Syndrome Bethesda: National Institute of Health; 2008.

Rodriguez, J., Cabal, A., Síndrome de Dandy-Walker. Aten Primaria, 2010; 42 (1): 50-51

Spennato P, Mirone G, Nastro A. Hydrocephalus in Dandy- Walker malformation. Child's Nervous System 2011;27:10, 1665-81.

Treviño M, González N., Montes J., García J., Martínez H., Rivera G.. Malformación de Dandy-Walker. Arch Argent Pediatr 2014;112(1):103-105

Wald M, Lowrenz K, Deutinger J, Weninger M. Verifications of anomalies of the central nervous system delected by prenatal ultrasound. Ultraschall in Med 2004; 25(3) :214-7. 
Zuluaga, H., Henao, K., Herrera, Y., Mejía, M., Prisco, L., Malformación de Dandy-Walker: reporte de un caso. Colombia. 2009. Medicina UPB 28(2): 147-153. 